\title{
Performance of mid-upper arm circumference to diagnose acute malnutrition in a cross-sectional community-based sample of children aged 6-24 months in Niger
}

\author{
Sarah K Marshall1 ${ }^{1 \S}$, Joel Monárrez-Espino ${ }^{1,2}$ and Anneli Eriksson ${ }^{1}$ \\ 'Department of Public Health Sciences, Karolinska Institutet, Solnavägen 1, 17177 Solna, Sweden \\ ${ }^{2}$ Coordination for Health Research. Christus LatAm Hub - Center for Excellence and Innovation, 66260 Monterrey, Nuevo León, Mexico
}

BACKGROUND/OBJECTIVES: Accurate, early identification of acutely malnourished children has the potential to reduce related child morbidity and mortality. The current World Health Organisation (WHO) guidelines classify non-oedematous acute malnutrition among children under five using Mid-Upper Arm Circumference (MUAC) or Weight-for-Height Z-score (WHZ). However, there is ongoing debate regarding the use of current MUAC cut-offs. This study investigates the diagnostic performance of MUAC to identify children aged 6-24 months with global (GAM) or severe acute malnutrition (SAM).

SUBJECTS/METHODS: Cross-sectional, secondary data from a community sample of children aged 6-24 months in Niger were used for this study. Children with complete weight, height and MUAC data and without clinical oedema were included. Using WHO guidelines for GAM (WHZ $<-2$, MUAC $<12.5 \mathrm{~cm}$ ) and SAM $(\mathrm{WHZ}<-3$, MUAC $<11.5 \mathrm{~cm})$, the sensitivity (Se), specificity (Sp), predictive values, Youden Index and Receiver Operating Characteristic (ROC) curves were calculated for MUAC when compared with the WHZ reference criterion.

RESULTS: Of 1161 children, $23.3 \%$ were diagnosed with GAM using WHZ, and $4.4 \%$ with SAM. Using current WHO cut-offs, the Se of MUAC to identify GAM was greater than for SAM (79 vs. 57\%), yet the Sp was lower (84 vs. $97 \%$ ). From inspection of the ROC curve and Youden Index, Se and Sp were maximised for MUAC $<12.5 \mathrm{~cm}$ to identify GAM (Se 79\%, Sp $84 \%$ ), and MUAC < $12.0 \mathrm{~cm}$ to identify SAM (Se $88 \%$, Sp $81 \%$ ).

CONCLUSIONS: The current MUAC cut-off to identify GAM should continue to be used, but when screening for SAM, a higher cut-off could improve case identification. Community screening for SAM could use MUAC $<12.0 \mathrm{~cm}$ followed by appropriate treatment based on either MUAC $<11.5 \mathrm{~cm}$ or $\mathrm{WHZ}<-3$, as in current practice. While the practicalities of implementation must be considered, the higher SAM MUAC cut-off would maximise early case-finding of high-risk acutely malnourished children.

Nutrition Research and Practice 2019;13(3):247-255; https://doi.org/10.4162/nrp.2019.13.3.247; pISSN 1976-1457 elSSN 2005-6168

Keywords: Severe acute malnutrition, anthropometric measures, community screening, diagnosis, Niger

\section{INTRODUCTION}

Worldwide acute malnutrition or wasting (low weight-forheight) affects 50 million children aged under five years [1]. These children are vulnerable to short-term risks of disease, impaired development and mortality, and irreversible long-term risks such as short stature and impaired cognition. Undernutrition is estimated to be associated with $45 \%$ of the deaths among children under five every year, occurring mostly in lowand middle-income countries [2]. Identification of acutely malnourished children is thus a priority for timely treatment and ultimately to avoid child illness and death.

To measure non-oedematous acute malnutrition in children aged 6-59 months, the World Health Organisation (WHO) guidelines endorse the use of Mid-Upper Arm Circumference (MUAC) or Weight-for-Height Z-score (WHZ), using these to classify Severe Acute Malnutrition (SAM) (WHZ <-3, MUAC $<11.5 \mathrm{~cm}$ ), Moderate Acute Malnutrition (MAM) (WHZ -3 to $<-2$, MUAC 11.5 to $<12.5 \mathrm{~cm}$ ) and Global Acute Malnutrition (GAM) (WHZ <-2, MUAC < $12.5 \mathrm{~cm}$ ) [3,4].

So far, the WHZ indicator has become the accepted 'goldstandard' anthropometric indicator for acute malnutrition. WHZ is an index of weight and height based on the WHO 2006 Child Growth Standards [5]. It is used for diagnosis, but also to compute prevalence estimates and global indicators. Yet, to obtain the $\mathrm{WHZ}$, a measurement of height and weight by trained personnel using a board and scale is required. The results obtained can then be plotted using a growth chat.

\footnotetext{
This research received no specific grant funding. Resources, materials and publication costs were supported by Karolinska Institutet. The original research was funded by Médecins Sans Frontières (MSF), UNICEF and United Nations World Food Program. Epicentre holds the data and receives core funding from MSF. ${ }^{\S}$ Corresponding Author: Sarah K Marshall, Tel. 61-431-921-909, Email. sarah.kate.marshall@outlook.com Received: April 11, 2019, Revised: April 30, 2019, Accepted: May 9, 2019

This is an Open Access article distributed under the terms of the Creative Commons Attribution Non-Commercial License (http://creativecommons.org/licenses/by-nc/3.0/) which permits unrestricted non-commercial use, distribution, and reproduction in any medium, provided the original work is properly cited.
} 
In low-income, high-prevalence or emergency settings where resources may be limited, MUAC becomes an essential tool, as it is a quick, easy and cheap method to identify acutely malnourished children using only a non-stretch tape around the mid-upper arm. MUAC is primarily used to produce rapid prevalence estimates $[6,7]$, and for community screening via Community-based Management of Acute Malnutrition (CMAM) [8]. However, in spite of MUAC's higher functionally as an alternative in areas of scare resources, its diagnostic value continues to be debated.

Since the use of the 2006 WHO Growth Standards [5], the SAM MUAC cut-off was increased from 11.0 to $11.5 \mathrm{~cm}[3,9]$. Three subsequent studies investigating MUAC performance, suggest revised cut-offs for SAM (ranging from 11.8 to $13.7 \mathrm{~cm}$ ) [10-12] with one recommending age- and sex-specific MUAC cut-offs [10]. Three other performance studies concluded that MUAC should not be used alone as it fails to accurately identify acutely malnourished children according to WHZ [13-15].

Studies also predominantly found that the children identified with GAM or SAM using MUAC versus WHZ were not the same groups. This aligns with existing knowledge, as highlighted in the WHO Guidelines [3]. Children identified with MUAC tend to be female, younger and shorter for their age $[3,16,17]$. While this may be favourable for screening higher-risk children, it questions the value of MUAC to diagnose the individual child with acute malnutrition.

A recent work by Grellety et al. [18] showed large discrepancies when using MUAC and WHZ to identify GAM and SAM and the differences seen across countries. The authors highlighted the relevance of within country and regional variations of the indicators, and advised that both indicators should be used as independent admission criteria. Therefore, the inconsistent conclusions regarding the diagnostic value of current MUAC cut-offs to diagnose acute malnutrition along with the discrepancy seen across countries warrants continued investigation.

The present study was set in Niger, where the mortality rate of children under five is trending downward (326 deaths/1000 live births in 1990 to 114 in 2012), but still ranks among the highest in the world [19]. While there have been improvements in malnutrition prevalence, levels still remain high (children under five stunted $43.9 \%$ or wasted $18 \%$ ) [20]. Recognising the priority for acute malnutrition identification, two recent studies conducted in Niger showed that mothers can reliably use MUAC to diagnose their children as acutely malnourished [21,22]. With evidence for community monitoring using MUAC in Niger and the variability observed when using MUAC across countries, this study aimed at assessing the performance of MUAC as an indicator of GAM and SAM using WHZ as the gold-standard for the diagnosis of acute malnutrition among children aged 6-24 months in rural Niger.

\section{SUBJECTS AND METHODS}

\section{Study Design}

This study used cross-sectional, secondary data from a community-based sample of children aged 6 to $<24$ months living in rural Madarounfa, Niger. The data was collected during
August 2011 until October 2012. The current study used baseline data from Prudhon et al. [23] that originated from a larger study by Langendorf et al. [24]. The primary studies were conducted by Epicentre in partnership with national nongovernment organisation, Niger Health Forum (FORSANI). Full methodological details are published elsewhere [23,24]. The STROBE checklist [25] was used for the reporting of this cross-sectional study (Supplementary Table 1).

\section{Study Setting}

This study was set in Madarounfa, a rural district of the Maradi region of Niger. During the study period 2011 to 2012, the Maradi region was affected by drought and at risk of severe food insecurity [26]. Niger is a low-income country, ranks the lowest on the Human Development Index (Index 0.354, Rank, 189) [27], and it is prone to political instability, chronic food insecurity and natural crises.

\section{Study Sample}

Participants of this study were children aged 6 to $<24$ months with complete anthropometric data (weight, height, MUAC), and without clinical signs of oedema, as this can increase body weight and compromise the reliability of $\mathrm{WHZ}$ and MUAC. The participants were enrolled in the primary study based on eligibility criteria: height $>60$ and $\leq 80 \mathrm{~cm}$, nil known food allergies or difficulties swallowing, and primarily residing in one of the 17 selected study villages within the Madarounfa district.

\section{Data Collection}

All houses in the selected villages were visited and eligible children, after informed consent was given, had anthropometrics measured (height/length, weight, MUAC) by trained nutrition assistants according to standardised protocol and using calibrated equipment. Sex of the child, date-of-inclusion and birth-date were recorded using a questionnaire completed by the primary caregiver. If date-of-birth was unknown, a local calendar of events was used to assist the primary caregiver to recall the birth and estimate the child's age, as recommended by $\mathrm{WHO}[3]$.

\section{Data management}

Children with missing information for key variables: MUAC, height, weight or age were excluded from the analysis (Fig. 1). WHO Anthro software (version 3.2.2, January 2011) with macro for SPSS was used to calculate $z$-scores for anthropometric indices (Height-for-Age Z-score: $\mathrm{HAZ}$, and WHZ). Any children with extreme values for z-scores according to commonly applied WHO flag recommendations [28] were excluded from analysis (Fig. 1). Both MUAC and WHZ indicators were dichotomised according to the WHO guidelines for GAM (WHZ $<-2$ and MUAC $<12.5 \mathrm{~cm})$ and also for SAM $(\mathrm{WHZ}<-3$ and MUAC $<11.5 \mathrm{~cm}$ ).

Where age was obtained using a local events calendar, it was recorded to the nearest day. Where date-of-birth was known, date-of-inclusion was subtracted from date-of-birth and age was calculated in days. The age variable was dichotomized according to children aged 6-11.9 months and 12-23.9 months. This is of practical relevance as children aged 6-12 months have 


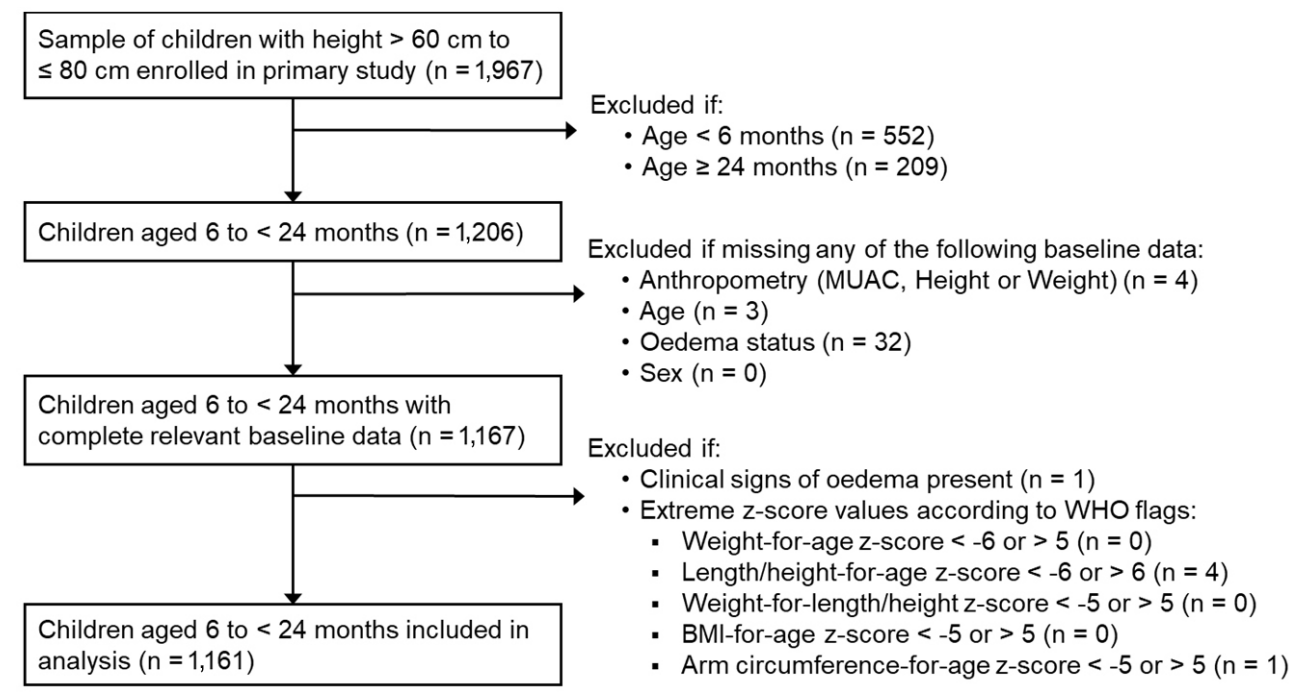

Fig. 1. Study design and eligibility criteria. MUAC, Mid-upper arm circumference; WHO, World Health Organisation

greater growth velocity and comparatively plateau at 12-24 months [29]. Secondly, feeding guidelines change between these age groups; while continued breastfeeding is recommended for both age groups, children 12-24 months require greater quantities and more complementary foods [30]. Stunting status was dichotomised as $\mathrm{HAZ}<-2$ stunted or $\mathrm{HAZ}$ $>-2$ not stunted, as per WHO growth standards [5].

\section{Statistical analysis}

SPSS software Version 23 (SPSS Statistics for Windows, Armonk, NY: IBM Corp) was used for analysis. Means and standard deviations (SD) were calculated for normallydistributed height, weight and MUAC variables. Frequencies of categorized standard $\mathrm{WHO}$ growth indicators were calculated (WHZ, HAZ and MUAC). These variables were stratified by age and sex for descriptive purposes.

Contingency tables for GAM and SAM identification with current MUAC and WHZ cut-offs were used to calculate sensitivity (Se), specificity (Sp), Positive Predictive Values (PPV) and Negative Predictive Values (NPV). These calculations were repeated when stratified by dichotomous variables: sex, age group and stunting status, factors known to differ with MUAC classification [16].

An empirical Receiver Operating Characteristic (ROC) analysis and Area Under the Curve (AUC) index was used to assess performance of MUAC as a diagnostic test when using $\mathrm{WHZ}$ as the criterion for acute malnutrition. The ROC analysis plots the Se and 1-Sp of the diagnostic test (MUAC) at intervals of cut-off points, against the diagnostic criteria WHZ. The analysis was run for diagnosis of GAM $(\mathrm{WHZ}<-2)$ and then SAM (WHZ $<-3)$.

The AUC obtained from ROC analysis, ranges between 0 and 1, where 0.5 signifies a worthless test ( $50 \%$ accuracy), and 1 signifies a perfect test (100\% accuracy). The upper and lower $95 \%$ Confidence Intervals (Cl) of AUC are also presented. Overlapping Cls for AUC were considered indicative of a lack of significant difference between stratified groups. The ROC analysis was repeated, stratified by age group, sex and stunting status.

Using the ROC analysis coordinates, the Youden Index [31] was calculated according to the formula $(\mathrm{Se}+\mathrm{Sp})-1$. Resultant values range between 0 and 1 , with 0 indicating no differential ability, and 1 indicating complete differential ability, giving equal weight to Se and Sp [32]. From inspection of the ROC graph and the Youden Index, the optimal cut-off points providing maximum $\mathrm{Se}$ and $\mathrm{Sp}$ were identified for GAM and SAM diagnosed by WHZ. If the optimal MUAC cut-off point differed from the current guidelines, MUAC was dichotomised using the new cut-off point and the Se, Sp, PPVs and NPVs were calculated using this value. Prevalence estimates of GAM and SAM using current guidelines for MUAC and $\mathrm{WHZ}$, and new MUAC cut-offs found through the ROC analysis are presented.

\section{Ethics approval}

For this secondary analysis, a Material Transfer Agreement was signed 13 June 2016 between Epicentre and Karolinska Institutet. Data was de-identified (no individual, cluster or village location could be identified). The primary study protocol received approval from National Ethical Committee of Niger (013/2011/CCNE), Ministry of Public Health and the Comite de Protection des Personnes, lle de France XI, France (11058) and was registered at ClinicalTrials.gov (NCT01828814).

\section{RESULTS}

\section{Sample characteristics}

A sample of 1161 children aged 6 to $<24$ months were included in the analysis. The median age of the children was 12.4 (inter-quartile range 7.6, 18.2) months, with $48 \%$ aged 6 to $<12$ months and $46 \%$ female. The mean height, weight and MUAC of the children in addition to categorised anthropometric indicators (WHZ, HAZ, and MUAC) categorised by current WHO classifications are presented in Table 1. Over half of the children were stunted (HAZ <-3 28.6\%, HAZ -3 to $<-234.3 \%$ ) and almost one quarter had low weight for their height $(\mathrm{WHZ}<-34.4 \%$, 
Table 1. Descriptive summary of anthropometric measures and indicators for the total sample and stratified by sex and age ( $\mathrm{n}=1,161$ ).

\begin{tabular}{|c|c|c|c|c|c|}
\hline & \multicolumn{2}{|c|}{ Sex } & \multicolumn{2}{|c|}{ Age in months } & \multirow{2}{*}{$\begin{array}{c}\text { Total } \\
(n=1,161)\end{array}$} \\
\hline & $\begin{array}{c}\text { Male } \\
(n=537)\end{array}$ & $\begin{array}{l}\text { Female } \\
(n=624)\end{array}$ & $\begin{array}{c}6-12 \\
(n=557)\end{array}$ & $\begin{array}{c}12-24 \\
(n=604)\end{array}$ & \\
\hline \multicolumn{6}{|l|}{ Anthropometric Measures } \\
\hline Height (cm) & $70.2 \pm 5.6$ & $68.9 \pm 5.9$ & $64.7 \pm 3.4$ & $73.9 \pm 3.7$ & $69.5 \pm 5.8$ \\
\hline Weight (kg) & $7.6 \pm 1.3$ & $7.1 \pm 1.3$ & $6.5 \pm 0.9$ & $8.1 \pm 1.0$ & $7.3 \pm 1.3$ \\
\hline MUAC (cm) & $13.2 \pm 1.1$ & $12.9 \pm 1.1$ & $12.8 \pm 1.1$ & $13.2 \pm 1.1$ & $13.0 \pm 1.1$ \\
\hline \multicolumn{6}{|l|}{ Anthropometric Indicators ${ }^{1)}$} \\
\hline \multicolumn{6}{|l|}{ Weight for Height Z-score, n (\%) } \\
\hline$<-3(\mathrm{SAM})$ & $33(6.1)$ & $18(2.9)$ & $27(4.8)$ & $24(4.0)$ & $51(4.4)$ \\
\hline-3 to $<-2$ (MAM) & $116(21.6)$ & $104(16.7)$ & $99(17.8)$ & $121(20.0)$ & $220(18.9)$ \\
\hline$<-2$ (GAM) & $149(27.7)$ & $122(19.6)$ & $126(22.6)$ & $145(24.0)$ & $271(23.3)$ \\
\hline \multicolumn{6}{|l|}{ Height for Age Z-score, n (\%) } \\
\hline$<-3$ (Severe stunting) & $201(37.4)$ & $131(21.0)$ & $138(24.8)$ & $194(32.1)$ & $332(28.6)$ \\
\hline-3 to $<-2$ (Moderate stunting) & $167(31.1)$ & $231(37.0)$ & $181(32.5)$ & $217(35.9)$ & $398(34.3)$ \\
\hline$<-2$ (Total stunting) & $368(68.5)$ & $362(58.0)$ & $319(57.3)$ & $411(68.0)$ & $730(62.9)$ \\
\hline \multicolumn{6}{|l|}{ MUAC, n (\%) } \\
\hline$<11.5$ cm (SAM) & $25(4.7)$ & $41(6.6)$ & $46(8.3)$ & $20(3.3)$ & $66(5.7)$ \\
\hline 11.5 to $<12.5 \mathrm{~cm}$ (MAM) & $119(22.2)$ & $176(28.2)$ & $157(28.2)$ & $138(22.8)$ & $295(25.4)$ \\
\hline$<12.5 \mathrm{~cm}(\mathrm{GAM})$ & $144(26.8)$ & $217(34.8)$ & $203(36.4)$ & $158(26.2)$ & $361(31.1)$ \\
\hline
\end{tabular}

MUAC, Mid-upper arm circumference; SAM, Severe Acute Malnutrition; SD, standard deviation; MAM, Moderate Acute Malnutrition; GAM, Global Acute Malnutrition

1) Anthropometric Indicators using current World Health Organisation guidelines for classification [5].

Table 2. Measures of diagnostic performance for MUAC $<12.5 \mathrm{~cm}$ to identify global acute malnutrition (GAM) ${ }^{11}$

\begin{tabular}{|c|c|c|c|c|c|c|c|c|}
\hline & \multirow{2}{*}{$\mathrm{Se}$} & \multirow{2}{*}{ Sp } & \multirow{2}{*}{ Youden Index } & \multirow{2}{*}{ PPV } & \multirow{2}{*}{ NPV } & \multirow{2}{*}{ AUC } & \multicolumn{2}{|c|}{ AUC 95\% Cl } \\
\hline & & & & & & & Lower & Upper \\
\hline Total & 0.79 & 0.84 & 0.63 & 0.60 & 0.93 & 0.89 & 0.87 & 0.91 \\
\hline \multicolumn{9}{|l|}{ Sex } \\
\hline Male & 0.73 & 0.91 & 0.64 & 0.76 & 0.90 & 0.91 & 0.89 & 0.94 \\
\hline Female & 0.87 & 0.78 & 0.65 & 0.49 & 0.96 & 0.91 & 0.88 & 0.93 \\
\hline \multicolumn{9}{|l|}{ Age } \\
\hline 6-12 months & 0.87 & 0.78 & 0.66 & 0.54 & 0.96 & 0.91 & 0.88 & 0.94 \\
\hline $12-24$ months & 0.72 & 0.89 & 0.61 & 0.67 & 0.91 & 0.90 & 0.88 & 0.93 \\
\hline \multicolumn{9}{|l|}{ Stunting status } \\
\hline Not stunted (HAZ >-2) & 0.66 & 0.91 & 0.57 & 0.61 & 0.93 & 0.90 & 0.86 & 0.94 \\
\hline Stunted (HAZ <-2) & 0.84 & 0.79 & 0.63 & 0.59 & 0.93 & 0.89 & 0.86 & 0.91 \\
\hline
\end{tabular}

Se, Sensitivity; Sp, Specificity; PPV, Positive Predictive Value; NPV, Negative Predictive Value; AUC, Area under the Curve; Cl, Confidence Interval; HAZ, Height for Age Z-score.

1) Contingency tables and ROC analysis were used to assess the diagnostic performance of MUAC $<12.5 \mathrm{~cm}$ to identify GAM when using WHZ as criterion according to WHO cut-offs (present $\mathrm{WHZ}<-2$ or not present $\mathrm{WHZ}<-2)$ in the total sample for children $(\mathrm{n}=1,161)$ and stratified by sex, age and stunting status.

WHZ 3 to $<-2$ 18.9\%).

\section{Diagnostic performance of MUAC}

A preliminary bivariate Pearson correlation of $\mathrm{WHZ}$ and MUAC variables showed a strong positive linear relationship $(R=0.759$, $P<0.001)$. The predictive values and empirical ROC analysis indicators for MUAC as a diagnostic test for GAM (WHZ <-2) and SAM $(\mathrm{WHZ}<-3)$ stratified by sex, age and stunting status, are presented in Tables 2 and 3. All AUC values were $>0.88$, and all $95 \%$ Cls within stratified groups overlapped, indicating no significant differences within stratified groups.

When MUAC $<12.5 \mathrm{~cm}$ was used to identify GAM (Table 2), the Se and Sp of MUAC $<12.5 \mathrm{~cm}$ ranged from 0.66 to 0.87 . For the total sample, Se was 0.79 (i.e. $79 \%$ true positive cases identified with both MUAC $<12.5$ and $\mathrm{WHZ}<-2$ ), Sp was 0.84 (i.e. $84 \%$ true negative cases, MUAC $>12.5$ and $\mathrm{WHZ}>-2$ ), Youden Index 0.63 and AUC was 0.89. The overlapping AUC Cls indicate no significant differences seen within each of the stratified categories. Among those who had a positive GAM screening test (MUAC $<12.5 \mathrm{~cm}$ ), the probability of GAM according to $\mathrm{WHZ}<-2$ criterion was $60 \%$ (PPV 0.60), whereas for a negative GAM screening test (MUAC $>12.5 \mathrm{~cm}$ ), the probability of not having GAM according to $\mathrm{WHZ}<-2$ criterion was higher (NPV 0.93).

When MUAC $<11.5 \mathrm{~cm}$ was used to identify SAM (Table 3), Se was lower than for GAM $(57 \%$ true positive cases, i.e. identified with both MUAC $<11.5 \mathrm{~cm}$ and $\mathrm{WHZ}<-3$ ) and $\mathrm{Sp}$ was higher; $97 \%$ children without SAM were accurately 
Table 3. Measures of diagnostic performance for MUAC $<11.5 \mathrm{~cm}$ to identify severe acute malnutrition $\left(\right.$ SAM) ${ }^{11}$

\begin{tabular}{|c|c|c|c|c|c|c|c|c|}
\hline & \multirow{2}{*}{ Se } & \multirow{2}{*}{ Sp } & \multirow{2}{*}{ Youden Index } & \multirow{2}{*}{ PPV } & \multirow{2}{*}{ NPV } & \multirow{2}{*}{ AUC } & \multicolumn{2}{|c|}{ AUC 95\% Cl } \\
\hline & & & & & & & Lower & Upper \\
\hline Total & 0.57 & 0.97 & 0.54 & 0.44 & 0.98 & 0.94 & 0.92 & 0.96 \\
\hline \multicolumn{9}{|l|}{ Sex } \\
\hline Male & 0.52 & 0.98 & 0.50 & 0.68 & 0.97 & 0.95 & 0.93 & 0.98 \\
\hline Female & 0.67 & 0.95 & 0.62 & 0.29 & 0.99 & 0.95 & 0.92 & 0.98 \\
\hline \multicolumn{9}{|l|}{ Age } \\
\hline 6-12 months & 0.67 & 0.95 & 0.61 & 0.39 & 0.98 & 0.94 & 0.91 & 0.97 \\
\hline $12-24$ months & 0.46 & 0.98 & 0.44 & 0.55 & 0.98 & 0.95 & 0.92 & 0.98 \\
\hline \multicolumn{9}{|l|}{ Stunting status } \\
\hline Not stunted (HAZ > -2) & 0.55 & 1.00 & 0.54 & 0.75 & 0.99 & 0.97 & 0.95 & 1.00 \\
\hline Stunted (HAZ <-2) & 0.58 & 0.95 & 0.52 & 0.40 & 0.98 & 0.92 & 0.89 & 0.96 \\
\hline
\end{tabular}

Se, Sensitivity; Sp, Specificity; PPV, Positive Predictive Value; NPV, Negative Predictive Value; AUC, Area under the Curve; Cl, Confidence Interval; HAZ, Height for Age Z-score.

1) Contingency tables and ROC analysis were used to assess the diagnostic performance of MUAC $<11.5 \mathrm{~cm}$ to identify SAM when using WHZ as criterion according to WHO cut-offs (present $\mathrm{WHZ}<-3$ or not present $\mathrm{WHZ}<-3)$ in the total sample for children $(n=1,161)$ and stratified by sex, age and stunting status.

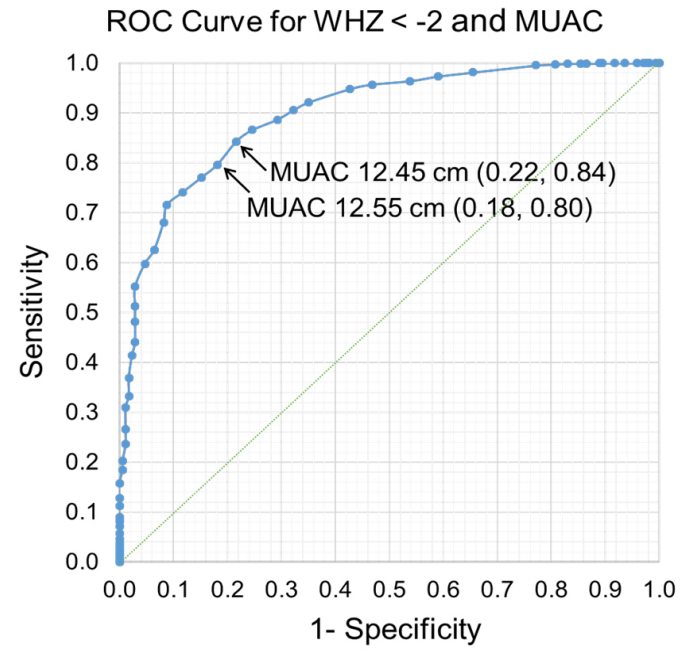

Fig. 2. Empirical receiver operating characteristic $(\mathrm{ROC})$ curve for diagnosis of GAM using MUAC. ROC analysis was performed with continuous test variable MUAC against dichotomous criterion variable WHZ (z-score <-2 or >-2) to identify GAM in the total sample, $n=1,161$ (AUC 0 89). GAM, Global Acute Malnutrition; MUAC, Mid-upper arm circumference; ROC, Receiver Operating Characteristic; WHZ, Weight-for-height z-score.

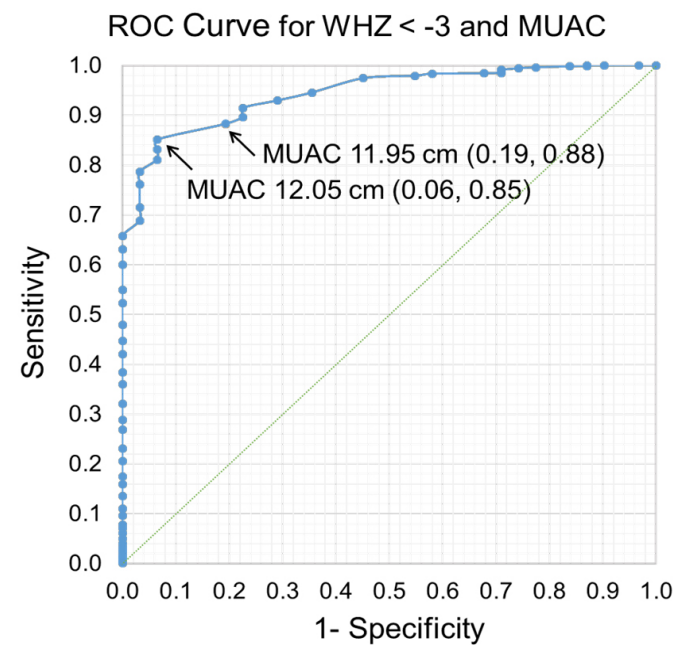

Fig. 3. Receiver operating characteristic (ROC) curve for diagnosis of SAM using MUAC. ROC analysis was performed with continuous test variable MUAC against dichotomous criterion variable $\mathrm{WHZ}$ (z-score $<-3$ or $>-3$ ) to identify SAM in the total sample, $n=1,161$ (AUC 0 94). MUAC, Mid-upper arm circumference; ROC, Receiver Operating Characteristic; SAM, Severe Acute Malnutrition; WHZ, Weight-for-height z-score.

\section{Prevalence of GAM and SAM identified using MUAC and WHZ}

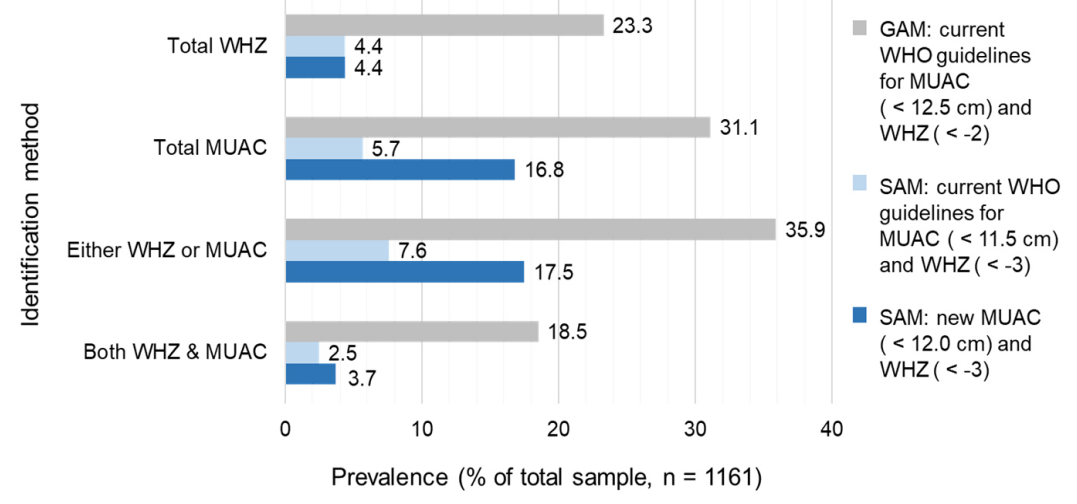

Fig. 4. Prevalence of GAM and SAM identified using current $W H O$ guidelines and MUAC $<12.0 \mathrm{~cm}$. MUAC $<12.0 \mathrm{~cm}$ identified from ROC analysis. 'Either WHZ or MUAC' denotes classification of acute malnutrition in one child using either of the indicators alone 'Both WHZ and MUAC' denotes classification of acute malnutrition of one child using both indicators. GAM, Global Acute Malnutrition; MUAC, Mid-upper arm circumference; ROC, Receiver Operating Characteristic; SAM, Severe Acute Malnutrition; WHZ, Weight-for-height Z-score. 


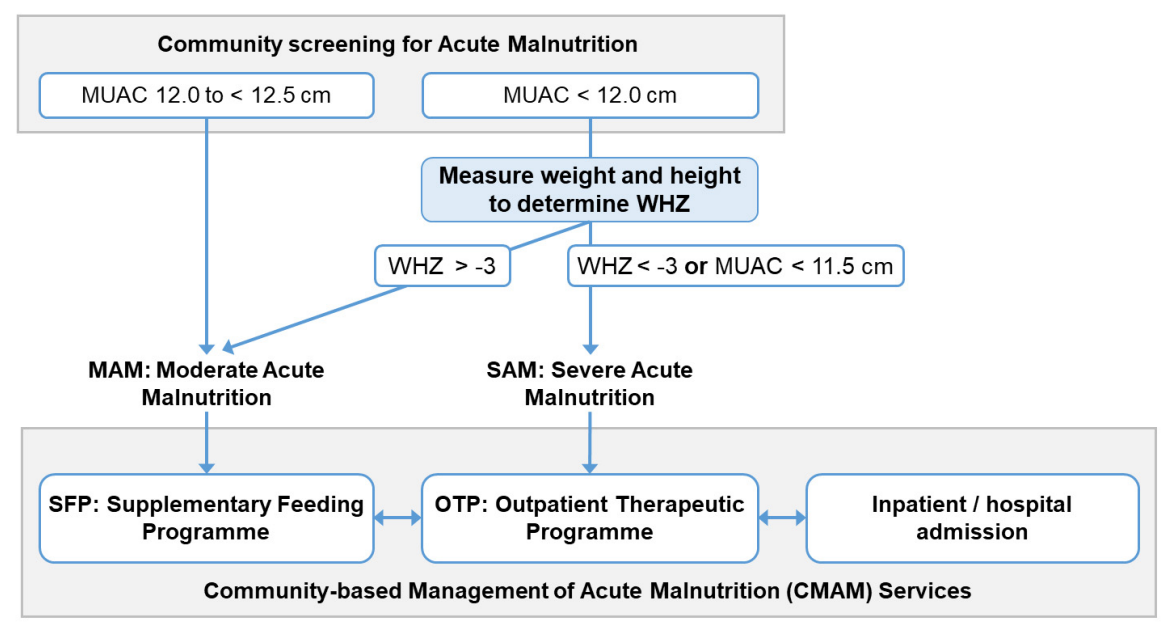

Fig. 5. Screening process for CMAM with SAM MUAC < $12.0 \mathrm{~cm}$. CMAM, Community-based Management of Acute Malnutrition; MUAC, Mid-upper arm circumference; SAM, Severe Acute Malnutrition; WHZ, Weight-for-height z-score.

identified (i.e. $97 \%$ true negative cases, MUAC $>11.5$ and $\mathrm{WHZ}$ $>-3)$. AUC showed greater strength (0.94), yet Youden Index was lower (0.54). Again, within each of the stratified categories there were no statistically significant differences. The most pronounced difference was seen for stunting status; MUAC was less sensitive but more specific among children with normal height for age when compared to stunted children. Compared with GAM, PPV for SAM was lower and NPV was higher $(0.44$ and 0.98 respectively).

\section{Receiver operating characteristic curves}

Upon inspection of the ROC curve (Fig. 2) and Youden Index calculations, the MUAC cut-off point maximising Se and Sp to identify GAM was approximately $12.5 \mathrm{~cm}$ (Se 0.79, Sp 0.84, Youden Index 0.63). Upon inspection of the ROC curve (Fig. 3) and Youden Index, the MUAC cut-off point maximising Se and Sp was approximately MUAC $12.0 \mathrm{~cm}$ (Se 0.84, Sp 0.87, Youden Index 0.71).

\section{Acute malnutrition prevalence estimates}

MUAC overestimated prevalence of GAM (MUAC 31.1\%, versus $\mathrm{WHZ} 23.3 \%$ ) and SAM (MUAC 5.7\% versus $\mathrm{WHZ} 4.4 \%$ ) (Fig. 4). If both indicators were applied, fewer children were identified with GAM (18.5\%) and with SAM (3.3\%). When using MUAC $<12.0 \mathrm{~cm}$, identified from the ROC analysis, there were greater true positives (3.7\% SAM identified with both $\mathrm{WHZ}$ and MUAC) and fewer cases were missed ( $0.7 \%$ identified with $\mathrm{WHZ}$ only), however the SAM prevalence was largely overestimated (16.8\%) (Fig. 4).

\section{DISCUSSION}

This study assessed the diagnostic performance of current MUAC cut-offs to identify acute malnutrition diagnosed using the gold-standard $\mathrm{WHZ}$ in a large community sample of children aged 6-24 months from rural Niger. Se and Sp of MUAC was optimised using the current cut-off for GAM, and a higher cut-off for SAM. Based on our findings, we recommend community screening using the current MUAC cut-off $<12.5$ $\mathrm{cm}$ for GAM, but using MUAC $<12.0 \mathrm{~cm}$ for SAM. Children identified at risk of SAM using MUAC $<12.0 \mathrm{~cm}$ should be referred to health care services and, as currently recommended, receive treatment based on $\mathrm{MUAC}<11.5 \mathrm{~cm}$ and $\mathrm{WHZ}<-3$ to achieve increased identification and accuracy, vital for timely management of SAM.

Within this sample, the current MUAC cut-off for SAM failed to capture $40 \%$ of severely malnourished children. Using the increased cut-off $<12.0 \mathrm{~cm}$ identified in this study, this figure decreased to $16 \%$ (Se $84 \%$, true-positive identification increased from $n=29$ to 43). Acknowledging the potential and valid criticism of placing equal importance on Se and $\mathrm{Sp}$, this objective method applied in this context achieved higher Se. This is arguably preferred, as more high-risk severely malnourished children would be appropriately referred to Outpatient Therapeutic Programmes (OTP) to receive treatment.

According to our results, using MUAC $<12.0 \mathrm{~cm}$ as a sole referral criterion would more than triple the number of children directed to OTP, and only one third of these would be true-positive SAM cases. Even with the increasing costeffectiveness of CMAM [33], this is not ideal in a resource- and financially-constrained environment. It would also place undue stress on children and families when SAM is not present (false-positive cases). To mitigate these issues, the authors support the two-step community screening process, first using MUAC $<12.0 \mathrm{~cm}$ for SAM, followed by weight and height measurements to make diagnoses using the $\mathrm{WHZ}$ indicator (illustrated in Fig. 5). Depending on local feasibility, WHZ assessment may take place at the location of community screening or at the local health service.

Other studies investigating the validity of current MUAC cut-offs to diagnose acute malnutrition also found low Se using the current MUAC cut-offs [10-15] and those assessing SAM highlighted the need to revise the MUAC $<11.5 \mathrm{~cm}$ cut-off [11-13,15]. Laillou et al. [11] suggest increasing the MUAC above the current cut-off to $<13.3 \mathrm{~cm}$ at screening and then using current MUAC and WHZ for SAM diagnosis, as per the procedure 
proposed in this study. Our study suggests a middle ground and we assume that the additional time, staff and equipment requirements of obtaining $\mathrm{WHZ}$ can be justified when the alternatives are either increasing OTP referrals by $300 \%$ using MUAC $<12.0 \mathrm{~cm}$, or continuing to miss $40 \%$ of children with SAM using the current MUAC cut-off.

In contrast to the findings of this study, two validity studies both obtained lower Se values and concluded MUAC $<12.5 \mathrm{~cm}$ should not be used as a stand-alone criterion for GAM [10,14]. Our diagnostic performance measures did not show significant differences when stratified by sex, age or stunting status. This may be partly explained by the younger age group and the high prevalence of stunting in our study sample. Differences in age are most apparent for 6-23 months compared with 24-59 months [3], and WHZ can be influenced by body shape and can affect prevalence of acute malnutrition in some populations [34].

It is accepted and highlighted in the WHO guidelines [3] that MUAC and WHZ indicators identify different groups of acutely malnourished children. These differences are more apparent for SAM, as seen in our results. If two-step screening using MUAC and then $\mathrm{WHZ}$ was implemented according to the current guidelines, approximately $40 \%$ of the children with SAM would be missed (prevalence using both indicators: $2.5 \%, \mathrm{WHZ}$ only: 4.4\%). This poor overlap of indicators for SAM provides support for our proposed change to MUAC of $<12.0 \mathrm{~cm}$ screening. In addition to the low cost and ease of using MUAC as the principal screening tool, it may be a better predictor of mortality for SAM in younger age groups [3,35]. Therefore, after MUAC $<12.0 \mathrm{~cm}$ screening, children identified by $M U A C<11.5 \mathrm{~cm}$ or $\mathrm{WHZ}$ $<-3$ should receive treatment. The same method was suggested by Laillou et al. [11] and is currently practiced by WHO Integrated Management of Childhood Illness (IMCI) [36].

An additional consideration for implementation is the degree of measurement error when the proposed cut-offs differ by 0.5 $\mathrm{cm}$. There are many knowledge gaps regarding the inter- and intra-rater reliability of both MUAC and WHZ [37-39]. While potentially more difficult to measure, the $0.5 \mathrm{~cm}$ difference modifies only the proposed referral to step 2 of screening with $\mathrm{WHZ}$, not the overall children identified as acutely malnourished. Two recent studies conducted in Niger demonstrated that mothers can reliably use MUAC to classify their children as acutely malnourished, and suggests MUAC done regularly by mothers should become the focal point for efforts to scale-up CMAM $[20,21]$. There is no screening tool that surpasses MUAC in terms of ease and practicality and, with use among community members to achieve ongoing monitoring, the resultant earlier detection and treatment of children with acute malnutrition can ultimately reduce child mortality.

This study is of importance within the current food insecurity crisis and high acute and chronic malnutrition across areas of western Africa. These findings can assist in adapting acute malnutrition screening processes and increasing identification of high-risk children with SAM. Implementing changes to the screening process must be considered in context. The ability to cope with increased demand on staff, resources and finances must be assessed. In neighbouring countries Burkino Faso and Chad, MSF currently consider these factors, adjusting MUAC thresholds for treatment admission according to contextual factors, for example, ongoing mortality, presence of other nutrition service providers, access and resources [40]. It is highly relevant to consider this in the Sahel region, and other regions with ongoing high prevalence of chronic and acute malnutrition.

The results of these analyses suggest that the prevalence of acute malnutrition (i.e. GAM and SAM) is relatively similar when MUAC or WHZ are used with the existing cut-offs, but that a MUAC of $<12.0 \mathrm{~cm}$ could improve case identification when screening for SAM. Thus, current MUAC cut-offs should continue use when rapid estimates are required for efficiently assessing the nutrition needs of a location, particularly in emergency settings as recommended [6,7].

A strength of the present study is the methodological standards adhered to during anthropometric data collection, crucial for validity studies [41]. Measures were collected at one single time-point, were in accordance with globally-accepted, standardised protocol and undertaken by trained nutrition assistants using calibrated equipment. Given the critical importance of sound methodologies in validity studies, and the question of reliability of the indicators, it is recommended that future sutures assess and report on inter- and intra- observer reliability of the anthropometric measures.

This study made efforts to exclude children with clinical signs of oedema, which can influence weight and thus $\mathrm{WHZ}$, leading to false-negative or false-positive results. The primary data did not include other potential factors evidenced to impact either indicator, such as body shape, injury or disability $[14,34,42]$ which could cause random or systematic classification errors.

In conclusion, this study contributes to the body of evidence regarding the performance of current MUAC cut-offs to identify acute malnutrition defined by $\mathrm{WHZ}$ and suggests that $\mathrm{a}$ higher MUAC cut-off $(<12.0 \mathrm{~cm})$ could improve accurate case identification of SAM when undertaking community screening among children under five. Children with MUAC $<12.0 \mathrm{~cm}$ should then be referred to health services for diagnosis and subsequent treatment based on MUAC $<11.5 \mathrm{~cm}$ and $\mathrm{WHZ}<$ -3 , as per current guidelines. Existing MUAC cut-offs for GAM and SAM should continue to be used for rapid prevalence estimates and resultant direction of humanitarian response, policy and nutritional programming.

The acute malnutrition and stunting prevalence in this sample of children aged 6-24 months living rurally in Niger were comparable to regional national estimates. Acknowledging that there are country variations in the different groups of acutely malnourished children that MUAC and WHZ indicators [18], and that MUAC and WHZ are impacted by factors such as body shape [9], the authors cannot claim global application but are confident that these results have good external validity, particularly within the Sahel region. These findings can assist in adapting acute malnutrition screening processes and increasing identification of high-risk children with SAM.

The use of MUAC as a fast, effective, low-cost screening tool is invaluable. When paired with the gold-standard $\mathrm{WHZ}$, a greater number of severely malnourished children can be directed to treatment services. Early identification and intervention should be a priority for management of acute malnutrition in emergency settings and globally, ultimately reducing acute malnutrition 
related child morbidity and mortality.

\section{ACKNOWLEDGEMENTS}

The authors would like to acknowledge and thank Epicentre for their cooperation and provision of the study data.

\section{CONFLICT OF INTEREST}

SKM, JME and AE declare that they have no potential conflicts of interests. $A E$ is employed by, and JME is affiliated to Karolinska Institutet. None of the authors received funding or specific salary for this study or publication.

\section{AUTHORS' CONTRIBUTIONS}

AE and SKM obtained the data from Epicentre and arranged the transfer agreement. SKM, JME and AE formulated the research question and designed the study. SKM analysed the data and wrote the first draft of the manuscript. JME and $A E$ contributed to the writing of the manuscript. All authors read and approved the final manuscript.

\section{ABBREVIATIONS}

AUC: Area Under the Curve

$\mathrm{Cl}$ : Confidence Interval

CMAM: Community-based Management of Acute Malnutrition IMCI: Integrated Management of Childhood Illness

MAM: Moderate Acute Malnutrition

MSF: Médecins Sans Frontières (Doctors Without Borders)

MUAC: Mid-Upper-Arm Circumference

NPV: Negative Predictive Value

FORSANI: Forum Sante Niger (Niger Health Forum)

GAM: Global Acute Malnutrition

HAZ: Height-for-age z-score

PPV: Positive Predictive Value

ROC: Receiver Operating Characteristic

SAM: Severe Acute Malnutrition

Se: Sensitivity

Sp: Specificity

UN: United Nations

UNICEF: United Nations Children's Fund

WHO: World Health Organisation

WHZ: Weight-for-height z-score

\section{ORCID}

Sarah K Marshall: https://orcid.org/0000-0002-1462-3087

Joel Monárrez-Espino: https://orcid.org/0000-0002-0695-5356

Anneli Eriksson: https://orcid.org/0000-0003-3154-7247

\section{REFERENCES}

1. United Nations Children's Fund, World Health Organization, World Bank Group. Levels and Trends in Child Malnutrition, UNICEF - WHO - World Bank Group Joint Child Malnutrition Estimates. Geneva: World Health Organization; 2015.
2. Black RE, Victora CG, Walker SP, Bhutta ZA, Christian $P$, de Onis $M$, Ezzati M, Grantham-McGregor S, Katz J, Martorell R, Uauy R; Maternal and Child Nutrition Study Group. Maternal and child undernutrition and overweight in low-income and middle-income countries. Lancet 2013;382:427-51.

3. World Health Organization, United Nations Children's Fund. WHO Child Growth Standards and the Identification of Severe Acute Malnutrition in Infants and Children: a Joint Statement. Geneva: World Health Organization; 2009.

4. World Health Organization. Guideline: Updates on the Management of Severe Acute Malnutrition in Infants and Children. Geneva: World Health Organization; 2013.

5. WHO Multicentre Growth Reference Study Group. WHO Child Growth Standards: Length/Height-for-Age, Weight-for-Age, Weightfor-Length, Weight-for-Height and Body Mass Index-for-Age: Methods and Development. Geneva: World Health Organization; 2006.

6. The Sphere Project. Minimum standards in food security and nutrition. In: The Sphere Project, editor. The Humanitarian Charter and Minimum Standards in Humanitarian Response. Geneva: The Sphere Project; 2011. p.139-238.

7. UNHCR The United Nations Refugee Agency. Nutrition needs assessment. In: UNHCR, editor. UNHCR Emergency Handbook. Geneva: UNHCR The United Nations Refugee Agency; 2015. p.296-8.

8. Valid International. Community management of acute malnutrition (CMAM) [Internet]. Oxford: Valid International; 2014 [cited 2019 March 12]. Available from: http://www.validinternational.org.

9. Fernández MA, Delchevalerie P, Van Herp M. Accuracy of MUAC in the detection of severe wasting with the new WHO growth standards. Pediatrics 2010;126:e195-201.

10. Fiorentino M, Sophonneary P, Laillou A, Whitney S, de Groot R, Perignon $M$, Kuong K, Berger J, Wieringa FT. Current MUAC cut-offs to screen for acute malnutrition need to be adapted to gender and age: the example of Cambodia. PLoS One 2016;11:e0146442.

11. Laillou A, Prak S, de Groot R, Whitney S, Conkle J, Horton L, Un SO, Dijkhuizen MA, Wieringa FT. Optimal screening of children with acute malnutrition requires a change in current $\mathrm{WHO}$ guidelines as MUAC and WHZ identify different patient groups. PLoS One 2014;9:e101159.

12. Talapalliwar MR, Garg BS. Diagnostic accuracy of mid-upper arm circumference (MUAC) for detection of severe and moderate acute malnutrition among tribal children in central India. Int J Med Sci Public Health 2016;5:1317-21.

13. Dasgupta R, Sinha D, Jain SK, Prasad V. Screening for SAM in the community: is MUAC a simple tool? Indian Pediatr 2013;50:154-5.

14. Roberfroid D, Huybregts $L$, Lachat $C$, Vrijens F, Kolsteren $P$, Guesdon B. Inconsistent diagnosis of acute malnutrition by weight-for-height and mid-upper arm circumference: contributors in 16 cross-sectional surveys from South Sudan, the Philippines, Chad, and Bangladesh. Nutr J 2015;14:86.

15. Tripathy JP, Sharma A, Prinja S. Is mid-upper arm circumference alone sufficient to identify severe acute malnutrition correctly? Indian Pediatr 2016;53:166-7.

16. Briend A, Khara T, Dolan C. Wasting and stunting-similarities and differences: policy and programmatic implications. Food Nutr Bull 2015;36:S15-23.

17. Myatt $M$, Khara T, Collins S. A review of methods to detect cases of severely malnourished children in the community for their admission into community-based therapeutic care programs. Food 
Nutr Bull 2006;27:S7-23.

18. Grellety E, Golden MH. Weight-for-height and mid-upper-arm circumference should be used independently to diagnose acute malnutrition: policy implications. BMC Nutr 2016;2:10.

19. UNICEF, WHO, World Bank, UN-DESA Population Division. Levels and Trends in Child Mortality 2015. Geneva: World Health Organization; 2015.

20. Institut National de la Statistique (INS) et ICF International. Enquête Démographique et de Santé et à Indicateurs Multiples du Niger 2012. Calverton (MA): INS et ICF International; 2013.

21. Blackwell N, Myatt M, Allafort-Duverger T, Balogoun A, Ibrahim A, Briend $A$. Mothers understand and can do it (MUAC): a comparison of mothers and community health workers determining mid-upper arm circumference in 103 children aged from 6 months to 5 years. Arch Public Health 2015;73:26.

22. Alé FG, Phelan KP, Issa H, Defourny I, Le Duc G, Harczi G, Issaley K, Sayadi S, Ousmane N, Yahaya I, Myatt M, Briend A, Allafort-Duverger $T$, Shepherd S, Blackwell N. Mothers screening for malnutrition by mid-upper arm circumference is non-inferior to community health workers: results from a large-scale pragmatic trial in rural Niger. Arch Public Health 2016;74:38.

23. Prudhon C, Langendorf $C$, Roederer T, Doyon S, Mamaty AA, Woi-Messe L, Manzo ML, de Pee S, Grais RF. Effect of ready-to-use foods for preventing child undernutrition in Niger: analysis of a prospective intervention study over 15 months of follow-up. Matern Child Nutr 2017;13.

24. Langendorf $C$, Roederer $T$, de Pee S, Brown D, Doyon S, Mamaty AA, Touré LW, Manzo ML, Grais RF. Preventing acute malnutrition among young children in crises: a prospective intervention study in Niger. PLoS Med 2014;11:e1001714.

25. Vandenbroucke JP, von Elm E, Altman DG, Gøtzsche PC, Mulrow CD, Pocock SJ, Poole C, Schlesselman JJ, Egger M; STROBE Initiative. Strengthening the reporting of observational studies in epidemiology (STROBE): explanation and elaboration. PLoS Med 2007;4:e297.

26. USAID Famine Early Warning Systems Network. Niger food security brief [Internet]. Washington, D.C.: USAID Famine Early Warning Systems Network; 2014 [cited 2019 January 10]. Available from: http://www.fews.net/west-africa/niger/food-security-brief/may-2014.

27. United Nations Development Programme. Human development index country profile Niger [Internet]. NewYork (NY): United Nations Development Programme; 2015 [cited 2019 April 4]. Available from: http://hdr.undp.org/en/countries/profiles/NER.

28. Crowe S, Seal A, Grijalva-Eternod C, Kerac M. Effect of nutrition survey 'cleaning criteria' on estimates of malnutrition prevalence and disease burden: secondary data analysis. PeerJ 2014;2:e380.

29. WHO Multicentre Growth Reference Study Group. WHO Child Growth Standards: Growth Velocity Based on Weight, Length and Head Circumference: Methods and Development. Geneva: World Health Organization; 2009.

30. World Health Organization. Infant and Young Child Feeding: Model Chapter for Textbooks for Medical Students and Allied Health Professionals. Geneva: World Health Organization; 2009.

31. Youden WJ. Index for rating diagnostic tests. Cancer 1950;3:32-5.

32. Fluss $R$, Faraggi $D$, Reiser $B$. Estimation of the Youden index and its associated cutoff point. Biom J 2005;47:458-72.

33. Park SE, Kim S, Ouma C, Loha M, Wierzba TF, Beck NS. Community management of acute malnutrition in the developing world. Pediatr Gastroenterol Hepatol Nutr 2012;15:210-9.

34. Myatt M, Duffield A, Seal A, Pasteur F. The effect of body shape on weight-for-height and mid-upper arm circumference based case definitions of acute malnutrition in Ethiopian children. Ann Hum Biol 2009;36:5-20.

35. Briend A, Maire B, Fontaine $O$, Garenne M. Mid-upper arm circumference and weight-for-height to identify high-risk malnourished under-five children. Matern Child Nutr 2012;8:130-3.

36. World Health Organization. Integrated Management of Childhood Illness (IMCl) Chart Booklet. Geneva: World Health Organization; 2014.

37. Mwangome MK, Berkley JA. The reliability of weight-for-length/ height $Z$ scores in children. Matern Child Nutr 2014;10:474-80.

38. Saeed HA, Mogendi JB, Akparibo R, Kolsteren P. Reliability of mid-upper arm circumference measurements taken by community health nurses. Curr Res Nutr Food Sci 2015;3:26-35.

39. Mwangome MK, Fegan G, Mbunya R, Prentice AM, Berkley JA. Reliability and accuracy of anthropometry performed by community health workers among infants under 6 months in rural Kenya. Trop Med Int Health 2012;17:622-9.

40. Phelan KPQ, Lanusse $C$, van der Kam S, Delchevalerie $P$, Avril $N_{t}$ Hanson K. Simplifying the response to childhood malnutrition: MSF's experience with MUAC-based (and oedema) programming. Field Exch 2015;50:108-16.

41. Rutjes AW, Reitsma JB, Di Nisio M, Smidt N, van Rijn JC, Bossuyt PM. Evidence of bias and variation in diagnostic accuracy studies. CMAJ 2006;174:469-76.

42. World Health Organization. Physical status: the use and interpretation of anthropometry [Internet]. Geneva: World Health Organization; 1995 [cited 2019 April 4]. Available from: http://www.who.int/ childgrowth/publications/physical_status/en. 